\title{
Factores de riesgo para bacteriemia por Pseudomonas aeruginosa resistente a carbapenémicos adquirida en un hospital colombiano
}

\author{
Sandra Liliana Valderrama ${ }^{1}$, Pedro Felipe González¹, María Alejandra Caro¹, \\ Natalia Ardila ${ }^{1}$, Beatriz Ariza², Fabián Gili, Carlos Álvarez' \\ 1 Grupo de Investigación en Enfermedades Infecciosas, Unidad de Infectología, Hospital Universitario San \\ Ignacio, Pontificia Universidad Javeriana, Bogotá, D.C., Colombia \\ 2 Grupo de Investigación en Enfermedades Infecciosas, Laboratorio Clínico, Sección de Microbiología, Hospital \\ Universitario San Ignacio, Bogotá, D.C., Colombia \\ 3 Departamento de Epidemiología Clínica y Estadística, Pontificia Universidad Javeriana, Bogotá, D.C., Colombia
}

Introducción. Las bacteriemias por Pseudomonas aeruginosa resistentes a carbapenémicos son un problema de salud pública por las pocas alternativas de tratamiento disponibles, el aumento de la estancia hospitalaria, los costos que genera y el aumento en el riesgo de mortalidad.

Objetivo. Evaluar los factores de riesgo de bacteriemia por $P$. aeruginosa resistente a carbapenémicos adquirida en el Hospital Universitario San Ignacio, durante el periodo comprendido entre enero de 2008 y junio de 2014.

Materiales y métodos. Se hizo un estudio de casos y controles. Los casos eran de pacientes que presentaban bacteriemia por $P$. aeruginosa resistente a carbapenémicos y los controles eran pacientes con $P$. aeruginosa sensible a este grupo de antibióticos. Se midieron variables como el uso de meropenem o ertapenem, la inmunosupresión y la neoplasia, y se determinaron la mortalidad y el tiempo de estancia hospitalaria.

Resultados. Se evaluaron 168 pacientes, 42 casos y 126 controles. En el modelo multivariado se encontraron los siguientes factores de riesgo relacionados con la bacteriemia por $P$. aeruginosa resistente a carbapenémicos y adquirida en el hospital: uso de nutrición parenteral (odds ratio, $\mathrm{OR}=8,28$; $\left.\mathrm{IC}_{95 \%} 2,56-26,79 ; \mathrm{p}=0\right)$, uso de meropenem (OR=1,15; IC $\left.\mathrm{C}_{95 \%} 1,03-1,28 ; \mathrm{p}=0,01\right)$ y uso de ciprofloxacina $\left(\mathrm{OR}=81,99 ; \mathrm{IC}_{95 \%} 1,14-5884 ; \mathrm{p}=0,043\right)$.

Conclusión. Para el control de la aparición de $P$. aeruginosa resistente a carbapenémicos, se deben fortalecer los programas de control de antimicrobianos, promover el uso prudente de carbapenémicos y quinolonas, y vigilar el uso adecuado de la nutrición parenteral.

Palabras clave: Pseudomonas aeruginosa, factores de riesgo, bacteriemia, nutrición parenteral, ciprofloxacina.

doi: http://dx.doi.org/10.7705/biomedica.v36i2.2784

Risk factors for hospital-acquired bacteremia due to carbapenem-resistant Pseudomonas
aeruginosa in a Colombian hospital

Introduction: Bacteremia due to Pseudomonas aeruginosa resistant to carbapenems is a public health problem due to the limitations it places on therapeutic options, as well as the increased time patients must spend in hospital, costs and the risk of mortality.

Objective: To evaluate the risk factors for presentation of bacteremia due to carbapenem-resistant $P$. aeruginosa acquired in the Hospital Universitario San Ignacio between January 2008 and June 2014.

Materials and methods: This was a case control study in which the case patients presented bacteremia due to $P$. aeruginosa resistant to carbapenems and the control group included patients with $P$. aeruginosa susceptible to this group of antibiotics. Variables such as the previous use of meropenem and ertapenem, immunosuppression and neoplasia were measured. Mortality and duration of hospital were also described.

Results: In all, 168 patients were evaluated, of which 42 were cases and 126 controls. Using a multivariate model, the risk factors related to bacteremia due to carbapenem-resistant $P$. aeruginosa acquired in hospital were the following: use of parenteral nutrition ( $\mathrm{OR}=8.28 ; 95 \% \mathrm{Cl}: 2.56-26.79 ; \mathrm{p}=0$ ); use of meropenem ( $\mathrm{OR}=1.15 ; 95 \% \mathrm{Cl}: 1.03-1.28 ; \mathrm{p}=0.01)$; and use of ciprofloxacin ( $\mathrm{OR}=81.99 ; 95 \%$ Cl: $1.14-5884 ; p=0.043)$.

\section{Contribución de los autores:}

Todos los autores participaron en el diseño del estudio, el análisis e interpretación de los resultados, y en la redacción y aprobación del manuscrito. 
Conclusion: In order to prevent the emergence of carbapenem-resistant $P$. aeruginosa, antimicrobial control programs should be strengthened by promoting the prudent administration of carbapenems and quinolones. The correct use of parenteral nutrition should also be monitored.

Key words: Pseudomonas aeruginosa, risk factors, parenteral nutrition, bacteremia, parenteral nutrition, ciprofloxacin.

doi: http://dx.doi.org/10.7705/biomedica.v36i2.2784

La infección por Pseudomonas aeruginosa, un bacilo Gram negativo móvil ampliamente distribuido en el medio ambiente, tiene importancia en el contexto epidemiológico actual debido a la emergencia de resistencia a los carbapenémicos en este microorganismo, lo que representa una amenaza para la salud pública mundial (1).

La adquisición de $P$. aeruginosa resistente a carbapenémicos puede ocurrir tanto por vía endógena como exógena (2). La adquisición exógena esta usualmente asociada a la transmisión por contacto con las manos contaminadas de los trabajadores de la salud y la contaminación de superficies ambientales o de dispositivos médicos por procesos inadecuados de limpieza y desinfección. En su estudio, Morgan, et al., reportaron que cuatro (22\%) de 18 habitaciones albergaban pacientes con infecciones por $P$. aeruginosa resistente a múltiples fármacos y cultivos ambientales positivos para $P$. aeruginosa con este perfil, así como una posibilidad de colonización de manos, guantes o batas de 17,4 \% después del contacto con el paciente (2). La transmisión endógena está determinada por la colonización de un germen sensible a múltiples fármacos que se transforma en un germen resistente por la presión del uso de antimicrobianos. Johnson, et al., determinaron este tipo de transmisión como la causa de adquisición de $P$. aeruginosa resistente a imipenem en unidades de cuidados intensivos en $19 \%$ de los casos (3).

Según diversos estudios a nivel internacional, entre 3 y $5 \%$ de todas las infecciones bacterianas, y hasta 28 a $38 \%$ de las bacteriemias causadas por microorganismos Gram negativos, son causadas por $P$. aeruginosa (4), con tasas de incidencia de 4,7 casos por 100.000 personas al año, especialmente en países en desarrollo (5).

\footnotetext{
Correspondencia:

Sandra Liliana Valderrama, Unidad de Infectología, Hospital Universitario San Ignacio, Carrera $7 \mathrm{~N}^{\circ}$ 40-62, segundo piso, Bogotá, D.C., Colombia

Teléfono fax: (571) 3232667

slvalderrama@husi.org.co

Recibido: 13/04/15; aceptado: 04/11/15
}

En Colombia, aproximadamente 4,9\% de las bacteriemias son originadas por $P$. aeruginosa, y el porcentaje de resistencia a meropenem en este microorganismo varía entre 22 y 30,5\%, con una tendencia anual al incremento en los aislamientos en unidades de cuidado intensivo $(6,7)$.

Las bacteriemias por $P$. aeruginosa resistente a carbapenémicos se asocian con aumento de la mortalidad, prolongación de la estancia hospitalaria y un incremento considerable en los costos de atención $(8,9)$. Las personas afectadas generalmente presentan varias enfermedades concomitantes y antecedentes de uso frecuente de tratamientos inadecuados, lo que agrava su pronóstico y favorece el aumento en las tasas de mortalidad (10). La dificultad creciente para encontrar alternativas de tratamiento eficaces contra patógenos como $P$. aeruginosa resistente a carbapenémicos, obliga a buscar e implementar medidas que permitan contener la expansión de este microorganismo (11).

El objetivo de este estudio fue determinar los factores de riesgo relacionados con la aparición de este microorganismo en un hospital universitario de tercer nivel, con el fin de implementar estrategias de prevención de futuras infecciones hospitalarias $y$ brotes.

\section{Materiales y métodos \\ Diseño y población}

Se llevó a cabo un estudio de casos y controles anidado en una cohorte histórica en el Hospital Universitario San Ignacio de Bogotá, un centro de referencia de enfermedades de alto costo de tercer nivel de complejidad, que cuenta con 350 camas, incluidas 32 en la unidad de cuidados intensivos.

Se incluyeron pacientes mayores de 18 años hospitalizados durante el periodo comprendido entre enero de 2008 y junio de 2014. Se definieron como casos aquellos pacientes que presentaban bacteriemia adquirida en el hospital por $P$. aeruginosa resistente a carbapenémicos de tipo II: meropenem, imipenem o doripenem, confirmada mediante, al menos, un hemocultivo positivo. Los 
pacientes de control presentaban bacteriemia adquirida en el hospital por $P$. aeruginosa sensible a carbapenémicos. Se excluyeron los pacientes que ingresaron al hospital con bacteriemias hospitalarias adquiridas en otra institución de salud diferente al Hospital Universitario San Ignacio.

La muestra se calculó con el programa Epidat 3.0 de la Organización Panamericana de la Salud, utilizando como referencia el estudio de Tuon, et al. (12) y asumiendo como factor de riesgo el uso previo de carbapenémicos con una razón de momios (odds ratio, OR) de 4,17, y una frecuencia esperada de uso de carbapenem en $69 \%$ de los casos y $35 \%$ de los controles.

Se seleccionaron tres controles por cada caso, con un intervalo de confianza de $95 \%$ y un poder de $80 \%$, lo que resultó en una muestra de 34 casos y 100 controles. Cada caso se pareó con tres controles que contaran con aislamientos hechos 90 días antes y 90 días después de la fecha del aislamiento del caso. En los casos con más de tres controles, estos se seleccionaron aleatoriamente.

Se analizaron las siguientes variables independientes: edad, sexo, servicio hospitalario, estancia hospitalaria, tiempo de estancia en la unidad de cuidados intensivos y en urgencias, tiempo de uso previo de antibióticos, uso previo de imipenem, ertapenem, meropenem, ciprofloxacina, cefepime o piperacilina-tazobactam, puntaje de APACHE II, uso de asistencia respiratoria mecánica, días de asistencia respiratoria mecánica, neoplasias (hematológicas o de órgano sólido), neutropenia, falla renal, cirugía previa, inmunosupresión, enfermedad pulmonar obstructiva crónica (EPOC), procedimientos invasivos, origen de la bacteriemia y nutrición parenteral. También, se evaluaron la idoneidad del tratamiento antibiótico empírico y la mortalidad.

La sensibilidad a meropenem o imipenem de $P$. aeruginosa se estableció mediante una concentración inhibitoria mínima (CIM) menor de $2 \mu \mathrm{g} /$ $\mathrm{ml}$ (13); la bacteriemia adquirida en el hospital se definió como una infección del torrente sanguíneo aparecida 48 horas después del ingreso del paciente a la institución (14); la neutropenia se definió como un conteo de neutrófilos absolutos en sangre menor de 500 o $1.000 \times 10^{\%} / \mathrm{L}$ y signos indicativos de su disminución en los días posteriores; se consideró que había habido uso de imipenem, ertapenem, meropenem, ciprofloxacina, cefepime o piperacilina-tazobactam, si el paciente los había recibido, al menos, durante 48 horas; la falla renal se determinó de acuerdo con los criterios RIFLE (Risk, Injury, Failure, Loss, End stage kidney disease) (15); el tratamiento antimicrobiano empírico se consideró adecuado cuando se utilizó, al menos, un agente antimicrobiano activo para el aislamiento de $P$. aeruginosa, según el antibiograma en las primeras 48 horas posteriores a la toma del cultivo.

\section{Análisis microbiológico}

Se utilizaron los reportes de la sección de microbiología del laboratorio clínico sobre aislamientos en sangre positivos para $P$. aeruginosa, confirmados mediante prueba de sensibilidad antimicrobiana con el método automatizado MicroScan ${ }^{\circledR}$, y mediante confirmación manual de la sensibilidad a carbapenémicos por el método de KirbyBauer de difusión en agar. Los aislamientos se clasificaron como sensibles o resistentes a carbapenémicos con base en los puntos de corte para carbapenémicos modificados en junio de 2010 y recomendados por el Clinical and Laboratory Standards Institute (CLSI).

\section{Análisis estadístico}

El análisis estadístico se hizo con el programa Stata $^{\circledR}$ (versión 13,0). Inicialmente se describieron todas las variables usando estadísticas univariadas adecuadas. Las variables categóricas nominales se describieron usando proporciones y porcentajes; las variables ordinales se describieron usando proporciones, y para las variables continuas se usaron las medias aritméticas y las medianas.

La fuerza de la asociación entre las variables dependientes y las independientes, se midió mediante la OR y se calcularon intervalos de confianza de $95 \%$. Para los análisis bivariado y multivariado se utilizaron modelos de regresión logística condicional. Para identificar las variables significativamente asociadas con $P$. aeruginosa multirresistente en el análisis bivariado, se empleó un valor de $p$ menor de 0,2. Las variables identificadas en esta etapa se incluyeron en el modelo multivariado mediante la técnica de selección de variables de regresión, con una probabilidad de entrada de 0,05. En el modelo final se incluyeron de manera forzosa las variables de APACHE II (como una medida de gravedad del paciente) y de estancia hospitalaria en la unidad de cuidados intensivos, las cuales, además de otras variables como la nutrición parenteral, se consideran factores de confusión. 


\section{Consideraciones éticas}

Al ser un estudio observacional, no se hicieron intervenciones en los pacientes, por lo que no hubo riesgos para su salud y no hubo necesidad de consentimiento informado. Se tomaron los datos de las historias clínicas para analizarlos y registrarlos en la base de datos diseñada de manera anónima. El estudio fue aprobado por los comités institucionales de investigación y ética.

\section{Resultados}

Se seleccionaron las bacteriemias de adultos clasificadas como hospitalarias y se aplicaron los criterios de inclusión y exclusión, para una selección final de 42 casos y 126 controles. En la figura 1 se observa la distribución de los casos de bacteriemias por $P$. aeruginosa adquiridas en el Hospital Universitario San Ignacio. De los aislamientos correspondientes a 2014, solo se incluyeron los registrados hasta el mes de junio. Sin contar el 2014, el menor número de casos por año fue de seis y el mayor fue de siete. No hubo brotes debidos a este microorganismo durante el periodo del estudio.

La mediana de edad de los pacientes incluidos fue de 62 años, con un rango de 19 a 91 años; el $53 \%$ de los pacientes eran de sexo masculino. La mediana de la estancia hospitalaria fue de 17 días, con un rango entre 3 y 161 días; el $59 \%$ ( $n=99)$ de los pacientes recibió atención en la unidad de cuidados intensivos. La bacteriemia pulmonar fue la más frecuente $(n=51 ; 30 \%)$, seguida por la gastrointestinal ( $n=49 ; 26 \%)$. Las enfermedades concomitantes más frecuentes fueron la falla renal $(n=67 ; 39,8 \%)$ y las neoplasias $(n=47 ; 28 \%)$.

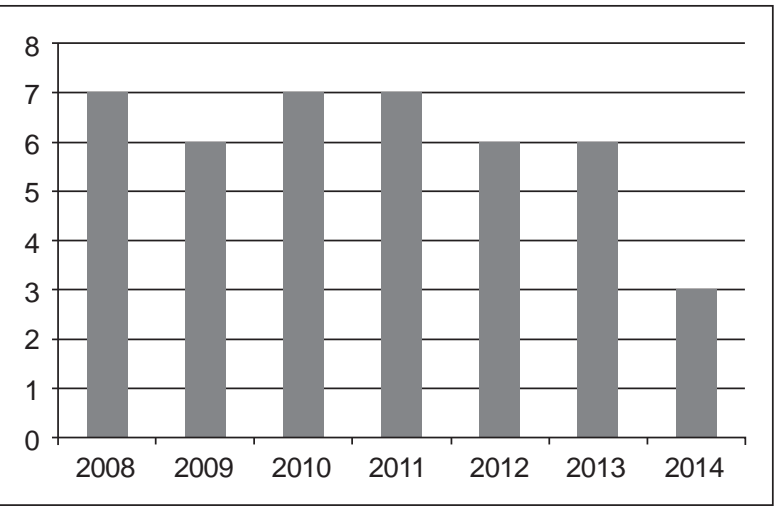

Figura 1. Casos de bacteriemia por Pseudomonas aeruginosa resistente a carbapenémicos adquirida en el Hospital Universitario San Ignacio, enero de 2008 a junio de 2014
En el análisis bivariado los factores de riesgo para la bacteriemia por $P$. aeruginosa resistente a carbapenémicos adquirida en el hospital con significación estadística, fueron la estancia hospitalaria, el tiempo de uso de antibióticos, el uso previo de meropenem, ciprofloxacina o cefepime, la cirugía previa y la nutrición parenteral (cuadro 1).

En el modelo multivariado se identificaron los siguientes factores de riesgo relacionados con la bacteriemia por $P$. aeruginosa resistente a carbapenémicos adquirida en el hospital: uso de nutrición parenteral $\left(\mathrm{OR}=8,28 ; \mathrm{IC}_{95 \%} 2,56-26,79\right.$; $\mathrm{p}<0,01)$, uso de meropenem $\left(\mathrm{OR}=1,15 ; \mathrm{IC}_{95 \%} 1,03-\right.$ $1,28 ; p=0,01)$ y uso de ciprofloxacina (OR=81,99; $\mathrm{IC}_{95 \%}$ 1,14-58,84; $\mathrm{p}=0,043$ ) (cuadro 2).

El porcentaje de pacientes que recibió tratamiento antimicrobiano empírico adecuado, fue menor en los casos que en los controles $(p=0,07)$. La mortalidad durante la hospitalización fue mayor en los casos $(p=0,022)$.

\section{Discusión}

En el presente estudio los factores de riesgo relacionados con la bacteriemia por $P$. aeruginosa resistente a carbapenémicos adquirida en el hospital, fueron la nutrición parenteral $(O R=8,28 ; p=0)$, el uso de meropenem $(\mathrm{OR}=1,15 ; \mathrm{p}=0,01)$ y el uso de ciprofloxacina ( $O R=81,99 ; p=0,043)$.

El uso de carbapenémicos, específicamente meropenem, se ha descrito ampliamente como un factor de riesgo para inducir la resistencia (16-19), como se señala en un metaanálisis de reciente publicación en el que se lo determinó como un factor estadísticamente significativo $\left(\mathrm{OR}=7,09 ; \quad \mathrm{IC}_{95 \%}\right.$ 5,43-9,25) (20). Estos datos concuerdan con los hallazgos descritos en este estudio e indican que la transmisión endógena es un mecanismo frecuente de aparición de $P$. aeruginosa resistente a carbapenémicos. Se debe enfatizar la importancia de los programas de control de antimicrobianos y la vigilancia, para garantizar que estos antimicrobianos se usen de manera empírica exclusivamente en pacientes con factores de riesgo como, por ejemplo, el uso previo de ceftriaxona o ciprofloxacina (21). Además, el tratamiento deberá ajustarse cuando se pueda reducir el espectro antimicrobiano con base en el resultado de los cultivos (22).

Es importante resaltar que el uso de imipenem no se determinó como un factor de riesgo, probablemente porque en el hospital se usa poco; el doripenem no se incluyó en el análisis porque su 
Cuadro 1. Descripción de los factores de riesgo asociados a bacteriemia por Pseudomonas aeruginosa resistente a carbapenémicos

\begin{tabular}{|c|c|c|c|c|c|c|c|}
\hline \multirow[b]{2}{*}{ Edad (mediana) (rango) } & \multicolumn{2}{|c|}{$\begin{array}{l}\text { Casos } \\
(n=42)\end{array}$} & \multicolumn{2}{|c|}{$\begin{array}{c}\text { Controles } \\
(n=126)\end{array}$} & \multirow{2}{*}{$\begin{array}{c}\text { OR } \\
0,995\end{array}$} & \multirow{2}{*}{$\begin{array}{c}\text { IC }_{95 \%} \\
0,98-1,01\end{array}$} & \multirow{2}{*}{$\begin{array}{c}\mathbf{p} \\
0,545\end{array}$} \\
\hline & 60 & $22-88$ & 64,5 & $19-91$ & & & \\
\hline \multicolumn{8}{|l|}{ Sexo $(n)(\%)$} \\
\hline Masculino & 25 & 59,5 & 64 & 50,8 & 1 & - & - \\
\hline Femenino & 17 & 40,5 & 62 & 49,2 & 1,41 & $0,70-2,83$ & 0,338 \\
\hline \multicolumn{8}{|l|}{ Servicio (n) (\%) } \\
\hline Piso & 14 & 33,3 & 46 & 36,5 & 1 & - & - \\
\hline $\mathrm{UCl}$ & 26 & 61,9 & 73 & 57,9 & 1,18 & $0,55-2,53$ & 0,692 \\
\hline Urgencias & 2 & 4,7 & 7 & 5,6 & 0,91 & $0,16-5,19$ & 0,918 \\
\hline Estancia hospitalaria en días (mediana) (rango) & 26 & 3-115 & 16 & $1-161$ & 1,01 & $1,00-1,03$ & 0,027 \\
\hline $\begin{array}{l}\text { Estancia hospitalaria en UCl en días } \\
\text { (mediana) (rango) }\end{array}$ & 12,5 & $0-106$ & 8 & $0-161$ & 1,01 & $0,99-1,03$ & 0,182 \\
\hline $\begin{array}{l}\text { Días de estancia hospitalaria en urgencias } \\
\text { (mediana) (rango) }\end{array}$ & 1 & $0-10$ & 1 & $0-14$ & 1,00 & $0,84-1,22$ & 0,923 \\
\hline Días de uso de antibióticos* (mediana) (rango) & 16,5 & $0-66$ & 7 & $0-71$ & 1,06 & $1,03-1,09$ & $<0,0001$ \\
\hline Uso previo de meropenem* (n) (\%) & 16 & 38,1 & 14 & 11,1 & 5,18 & $2,09-12,84$ & $<0,0001$ \\
\hline Uso previo de imipenem* (n) (\%) & 4 & 9,5 & 4 & 3,2 & 3,48 & $0,76-15,90$ & 0,107 \\
\hline Uso previo de ertapenem* (n) (\%) & 4 & 9,5 & 4 & 3,2 & 3,48 & $0,76-15,90$ & 0,107 \\
\hline Uso previo de ciprofloxacina* (n) (\%) & 5 & 11,9 & 1 & 0,8 & 15 & $1,75-128,39$ & 0,013 \\
\hline Uso previo de cefepime* (n) (\%) & 17 & 40,5 & 32 & 25,4 & 1,09 & $1,02-1,17$ & 0,013 \\
\hline Uso previo de piperacilina-tazobactam* $(n)(\%)$ & 14 & 33,3 & 26 & 20,7 & 1,95 & $0,89-4,26$ & 0,096 \\
\hline Puntaje en Apache II (mediana, rango & 14,5 & $0-29$ & 11,5 & $0-33$ & 1,04 & $0,99-1,10$ & 0,107 \\
\hline Uso de asistencia respiratoria (n) (\%) & 28 & 66,7 & 79 & 62,7 & 1,18 & $0,57-2,47$ & 0,647 \\
\hline Días con asistencia respiratoria (mediana) (rango) & 9 & $0-63$ & 6 & $0-144$ & 1 & $0,99-1,03$ & 0,366 \\
\hline \multicolumn{8}{|l|}{ Neoplasia (n) (\%) } \\
\hline No & 29 & 69,1 & 92 & 73 & 1 & - & - \\
\hline Hematológica & 5 & 11,9 & 14 & 11,1 & 1,13 & $0,38-3,36$ & 0,825 \\
\hline En órgano sólido & 8 & 19 & 20 & 15,9 & 1,27 & $0,51-3,19$ & 0,612 \\
\hline Neutropenia (n) (\%) & 8 & 6,4 & 5 & 11,9 & 2,05 & $0,61-6,90$ & 0,245 \\
\hline \multicolumn{8}{|l|}{ Falla renal $(n)(\%)$} \\
\hline No & 25 & 59,5 & 76 & 60,3 & 1 & - & - \\
\hline Aguda & 11 & 26,2 & 37 & 29,4 & 0,89 & $0,40-1,96$ & 0,77 \\
\hline Crónica & 5 & 11,9 & 9 & 7,1 & 1,65 & $0,52-5,21$ & 0,39 \\
\hline Diálisis & 1 & 2,4 & 4 & 3,2 & 0,78 & $0,09-7,2$ & 0,827 \\
\hline Cirugía previa (n) (\%) & 24 & 57,1 & 43 & 34,1 & 2,63 & $1,26-5,51$ & 0,01 \\
\hline Inmunosupresión (n) (\%) & 14 & 33,3 & 27 & 21,4 & 1,83 & $0,85-3,96$ & 0,124 \\
\hline $\operatorname{EPOC}(n)(\%)$ & 9 & 21,4 & 28 & 22,2 & 0,95 & $0,41-2,24$ & 0,914 \\
\hline Procedimientos invasivos (n) (\%) & 29 & 69,1 & 80 & 63,5 & 1,36 & $0,59-3,11$ & 0,469 \\
\hline \multicolumn{8}{|l|}{ Origen de la bacteriemia (n) (\%) } \\
\hline Primaria & 3 & 7,1 & 20 & 15,9 & 1 & - & - \\
\hline Pulmonar & 14 & 33,3 & 37 & 29,4 & 2,6 & $0,66-10,3$ & 0,173 \\
\hline Catéter & 0 & 0 & 7 & 5,6 & 0 & - & - \\
\hline Urinaria & 4 & 9,5 & 13 & 10,3 & 2,06 & $0,39-11,14$ & 0,402 \\
\hline Sistema gastrointestinal & 13 & 31 & 31 & 24,6 & 2,81 & $0,71-16,33$ & 0,14 \\
\hline Otras & 8 & 9,1 & 18 & 14,2 & 3,41 & $0,71-16,33$ & 0,124 \\
\hline Nutrición parenteral & 13 & 31 & 9 & 7,2 & 5,65 & $2,12-15,04$ & 0,001 \\
\hline Tratamiento empírico adecuado (n) y (\%) & 22 & 52,4 & 95 & 75,4 & & & 0,07 \\
\hline Mortalidad (n) (\%) & 24 & 57,1 & 45 & 35,7 & & & 0,022 \\
\hline
\end{tabular}

UCI: unidad de cuidado intensivo; EPOC: enfermedad pulmonar obstructiva crónica

*: solo se tuvieron en cuenta los días de uso de antibióticos previo al aislamiento de $P$. aeruginosa.

uso se introdujo en el hospital solo en el 2012 y es infrecuente. En varios estudios contemporáneos se ha evaluado el impacto del uso de ertapenem en la emergencia de microorganismos resistentes $y$, aunque los resultados son contradictorios, la mayoría de los hallazgos apuntan a que no hay asociación $(12,23)$. En el estudio de Sousa, et al., su empleo se relacionó con un menor uso de imipenem y con la mayor sensibilidad de $P$. aeruginosa a este medicamento (24). 
Cuadro 2. Modelo multivariado ( $n=168$ observaciones, 42 casos y 126 controles)

\begin{tabular}{lccl}
\hline Variable & OR & $\mathbf{p}$ & IC $_{95 \%}$ \\
\hline APACHE II & 1,02 & 0,58 & $0,95-1,09$ \\
Hospitalización en la unidad & 0,95 & 0,031 & $0,92-1$ \\
de cuidados intensivos & & & \\
Estancia hospitalaria & 1,02 & 0,33 & $0,98-1,05$ \\
Nutrición parenteral & 8,28 & $<0,001$ & $2,56-26,79$ \\
Uso previo de meropenem & 1,15 & 0,01 & $1,03-1,28$ \\
Uso previo de ciprofloxacina & 81,99 & 0,043 & $1,14-5884$ \\
\hline
\end{tabular}

El uso de quinolonas fue otro factor de riesgo estadísticamente significativo en este estudio, al igual que en otras investigaciones y en un metaanálisis (OR=2,73; $\left.\quad \mathrm{IC}_{95 \%} \quad 1,27-5,87\right) \quad$ (25). Se ha observado que las concentraciones inhibitorias de ciprofloxacina crean una presión de selección a favor de mutantes con un incremento en la expresión del adenosín monofosfato (AMP) cíclico (26), mientras que los niveles no inhibidores incrementan la frecuencia de la mutación de resistencia a carbapenémicos mediante el aumento de la regulación de la bomba de flujo MexEFOprM y a niveles reducidos de la porina OprD, lo que afecta especialmente a meropenem y, probablemente, es el mecanismo que mejor explica esta resistencia inducida (27). Las quinolonas no solo se han relacionado con la aparición de la resistencia de $P$. aeruginosa a los carbapenémicos, sino que también inducen la aparición de Staphylococcus aureus resistente a la meticilina y de la colitis por Clostridium difficile, por lo que su uso debe restringirse a los casos en los cuales sean la única elección debido a la alergia del paciente o por su buena penetración en los tejidos (28-30).

En el presente estudio se determinó el uso de la nutrición parenteral como un factor de riesgo para la bacteriemia por $P$. aeruginosa resistente a carbapenémicos adquirida en el hospital, con una OR elevada, de 8,28. La nutrición parenteral se ha relacionado con un mayor riesgo de bacteriemias en los pacientes, que varía entre 1,3 y $39 \%$, aunque los microorganismos generalmente relacionados han sido los cocos Gram positivos y las levaduras $(31,32)$.

Se ha propuesto a la hiperglucemia como una probable causa del aumento de las bacteriemias (33); sin embargo, en un estudio de cohorte, Dissanaike, et al. (34), no encontraron relación entre bacteriemia e hiperglucemia, pero sí una relación estadísticamente significativa cuando se aumentaba el aporte calórico a los pacientes, por lo que recomendaron vigilar este aspecto e, incluso, evitarlo aún por periodos cortos.

Otro estudio de cohorte evidenció que el riesgo de bacteriemia asociado a la nutrición parenteral era menor cuando la nutrición se recibía en una bolsa multicámara comercial, que si se suministraba en su forma preparada en farmacia $(19,6 \% \mathrm{Vs}$. $25,9 \% ; p<0,001$ ) (35). Estos factores pudieron haber causado un aumento en el riesgo de bacteriemia en el grupo de pacientes evaluados en el presente estudio, pero, al no haber sido evaluados de forma específica, solo pueden plantearse como hipótesis que explicarían el hallazgo e invitan a vigilar estos aspectos en los pacientes que reciben nutrición parenteral.

El ingreso en la unidad de cuidados intensivos se asoció con un incremento del riesgo de presentar bacteriemia de origen hospitalario de hasta 7,4 veces más que en otros servicios hospitalarios (25); esto sugiere que la estancia en la unidad de cuidados intensivos es un factor de riesgo para la infección por gérmenes multirresistentes (2). Sin embargo, en el presente estudio la estancia en la unidad de cuidados intensivos, prolongada o no, no se relacionó con la aparición de bacteriemia hospitalaria por $P$. aeruginosa resistente a carbapenémicos, probablemente porque las medidas institucionales de control de infecciones, como la supervisión de higiene de manos y de los aislamientos, se centraron en este servicio durante el periodo de estudio.

Otra de las variables evaluadas fue el puntaje de APACHE II (OR=1,02; IC $\left.{ }_{95 \%} 0,95-1,09 ; p=0,58\right)$, el cual no fue un factor de riesgo estadísticamente significativo tal vez porque constituye un factor de pronóstico más que uno de riesgo para la aparición de $P$. aeruginosa resistente a carbapenémicos. Como lo explican Jeong, et al. (8), su relación con una mayor mortalidad se debe, entre otras causas, a la propiedad innata de la elastasa de $P$. aeruginosa de alterar la estructura del tejido conjuntivo del huésped, y la quimiotaxis e interacción adecuada entre diferentes moléculas (IFN- $\gamma$, TNF-a, IgG, $\lg \mathrm{A}$, entre otras), lo que ocasiona una inadecuada reacción del huésped ante la infección, empeora la historia natural de la enfermedad, aumenta el puntaje de APACHE e implica un mayor riesgo de letalidad en el paciente.

En este estudio, el tratamiento antibiótico fue apropiado en $52,4 \%$ de los casos y en $75,4 \%$ de los controles, con una diferencia estadísticamente 
significativa, lo que en parte puede explicar la mayor mortalidad registrada entre los casos (57\% Vs. 35,7\%; $p=0,022)$. La bacteriemia de origen hospitalario por $P$. aeruginosa genera una mayor mortalidad que otras bacteriemias por Gram negativos que afectan a la tercera parte de los pacientes en las primeras 48 horas, con un valor neto entre 35 y $60 \%(10,36)$. En un estudio colombiano se encontró una mortalidad global por bacteriemia por $P$. aeruginosa de $43,9 \%$ y uno de los factores protectores fue el tratamiento antimicrobiano adecuado (37).

Entre las debilidades de la investigación debe mencionarse que, al tratarse de un estudio retrospectivo de casos y controles, no fue posible concluir que existía una relación causal perfecta entre los factores de riesgo determinados y la aparición de la bacteriemia por $P$. aeruginosa resistente a carbapenémicos (18). Por otro lado, debido al poco número de casos y al bajo poder, su precisión fue limitada, lo cual se evidenció en el intervalo de confianza para la OR de la ciprofloxacina en el modelo multivariado $\left(\mathrm{OR}=81,99 ; \mathrm{IC}_{95 \%} 1\right.$ 1,14-5884). Sin embargo, los factores de riesgo determinados fueron clínicamente relevantes y estadísticamente significativos, y otras variables del modelo, como el uso previo de meropenem, lograron estrechos intervalos de confianza (OR=1,15; $I C_{95 \%}$ 1,03-1,28).

Teniendo en cuenta que la transmisión exógena juega un papel crucial en la adquisición de $P$. aeruginosa, y que la contaminación ambiental es un factor crítico en su transmisión a los pacientes (28), otra limitación del estudio debida a su carácter retrospectivo fue no haberse evaluado factores asociados a una menor transmisión de la infección por $P$. aeruginosa resistente a carbapenémicos, como el cumplimiento de los protocolos de higiene de manos, el manejo de los aislamientos, y la limpieza y desinfección.

Para contrarrestar estas infecciones se debe priorizar, entonces, el control de gérmenes resistentes a carbapenémicos, específicamente la expansión de $P$. aeruginosa resistente a dichos medicamentos, haciendo énfasis en el fortalecimiento de los programas de control de antimicrobianos y del uso inadecuado de carbapenémicos y quinolonas, así como en el uso adecuado de la nutrición parenteral.

\section{Agradecimientos}

A Claudia Linares, por la revisión del manuscrito antes de la publicación.

\section{Conflictos de intereses}

Sandra Valderrama declara conflicto de intereses por haber recibido remuneración como conferencista de los laboratorios farmacéuticos Merck Sharp and Dhome, Pfizer, Glaxo, Janssen Cilag. Carlos Álvarez declara conflicto de intereses por haber recibido remuneración como conferencista de los laboratorios farmacéuticos Astra Zeneca y Pfizer. Pedro Felipe González, María Alejandra Caro, Natalia Ardila, Beatriz Ariza y Fabián Gil declaran no tener conflictos de intereses.

\section{Financiación}

La investigación fue financiada por el Hospital Universitario San Ignacio y la Pontificia Universidad Javeriana.

\section{Referencias}

1. Horino T, Chiba A, Kawano S, Kato T, Sato F, Maruyama, et al. Clinical characteristics and risk factors for mortality in patients with bacteriemia caused by Pseudomonas aeruginosa. Intern Med. 2012;51:59-64. http://dx.doi. org/10.2169/internalmedicine.51.5698

2. Morgan DJ, Rogawski E, Thom KA, Johnson JK, Perencevich EN, Shardell M, et al. Transfer of multidrugresistant bacteria to healthcare workers' gloves and gowns after patient contact increases with environmental contamination. Crit Care Med. 2012;40:1045-51. http://dx. doi.org/10.1097/CCM.0b013e31823bc7c8

3. Johnson JK, Smith G, Lee MS, Venezia RA, Stine OC, Nataro JP, et al. The role of patient-to-patient transmission in the acquisition of imipenem-resistant Pseudomonas aeruginosa colonization in the intensive care unit. J Infect Dis. 2009;200:900-5. http://dx.doi.org/10.1086/605408

4. Suárez $\mathbf{C}$, Peña $\mathbf{C}$. Influence of carbapenem resistance on mortality and the dynamics of mortality in Pseudomonas aeruginosa bloodstream infection. Int $\mathrm{J}$ Infect Dis. 2010;14s:e73-e8. http://dx.doi.org/10.1016/j.jijd.2009.11.019

5. Al-Hasan MN, Wilson JW, Lahr BD, Eckel-Passow JE, Baddour LM. Incidence of Pseudomonas aeruginosa bacteriemia: A population-based study. Am J Med. 2008;121:702-8. http://dx.doi.org/ 10.1016/j.amjmed.2008. 03.029

6. Hernández-Gómez C, Blanco VM, Motoa G, Correa A, Vallejo M, Villegas MV, et al. Evolución de la resistencia antimicrobiana de bacilos Gram negativos en unidades de cuidados intensivos en Colombia. Biomédica. 2014; 34(Suppl.1):91-100. http://dx.doi.org/10.7705/biomedica. v34i0.1667

7. Villa LM, Cortés JA, Leal AL, Meneses A, Meléndez MP, GREBO. Resistance to antibiotics in Pseudomonas aeruginosa in Colombian hospitals. Rev Chilena Infectol. 2013;30:605-10. http://dx.doi.org/10.4067/S0716-1018201 3000600005

8. Jeong SJ, Yoon SS, Bae IK, Jeong SH, Kim JM, Lee K. Risk factors for mortality in patients with bloodstream infections caused by carbapenem-resistant Pseudonoma 
aeruginosa: Clinical impact of bacterial virulence and strains on outcome. Diagn Microbiol Infect Dis. 2014;80:130-5. http://dx.doi.org/10.1016/j.diagmicrobio.2014.07.003

9. Tumbarello M, Repetto E, Treacarichi EM, Bernardini C, De Pascale G, Parisini A, et al. Multidrug-resistant Pseudomonas aeruginosa bloodstream infections: Risk factors and mortality. Epidemiol Infect. 2011;139:1740-9. http://dx.doi.org/10.1017/S0950268810003055

10. Micek ST, Lloyd AE, Ritchie DJ, Reichley RM, Fraser VJ, Kollef MH. Pseudomonas aeruginosa bloodstream infection: Importance of appropriate initial antimicrobial treatment. Antimicrob Agents Chemother. 2005;49:1306-11. http://dx.doi.org/10.1128/AAC.49.4.1306-1311.2005

11. Tacconelli E, Cataldo MA, Dancer SJ, De Angelis G, Falcone M, Frank U, et al. European Society of Clinical Microbiology. ESCMID guidelines for the management of the infection control measures to reduce transmission of multidrug-resistant Gram-negative bacteria in hospitalized patients. Clin Microbiol Infect. 2014;20(Suppl.1):1-55. http:// dx.doi.org/10.1111/1469-0691.12427

12. Tuon F, Gortz L, Rocha J. Risk factors for pan-resistant Pseudomonas aeruginosa bacteriemia and the adequacy of antibiotic therapy. Braz J Infect Dis. 2012;16:351-6. http://dx.doi.org/10.1016/j.bjid.2012.06.009

13. Clinical and Laboratory Standards Institute. Performance standards for antimicrobial susceptibility testing; approved standard. Twenty second information supplement. Document M100-S-22. Wayne: CLSI; 2012.

14. Hongsuwan M, Srisamang P, Kanoksil M, Luangasanatip $\mathbf{N}$, Jatapai A, Day NP, et al. Increasing incidence of hospital-acquired and healthcare-associated bacteriemia in northeast Thailand: A multicenter surveillance study. PLoS One. 2014;9:e109324. http://dx.doi.org/10.1371/journal.pone. 0109324

15. Bellomo R, Ronco C, Kellum JA, Mehta RL, Palevsky P. Acute Dialysis Quality Initiative workgroup. Acute renal failure - definition, outcome measures, animal models, fluid therapy and information technology needs: The Second International Consensus Conference of the Acute Dialysis Quality Initiative (ADQI) Group. Crit Care. 2004;8:R204-12. http://dx.doi.org/10.1186/cc2872

16. El Amari EB, Chamot E, Auckenthaler R, Pechère JC, van Delden C. Influence of previous exposure to antibiotic therapy on the susceptibility pattern of Pseudomonas aeruginosa bacteremic isolates. Clin Infect Dis. 2001;33: 1859-64. http://dx.doi.org/10.1086/324346

17. Falagas ME, Koletsi PK, Kopterides P, Michalopoulos A. Risk factors for isolation of strains susceptible only to polymyxin among patients with Pseudomonas aeruginosa bacteriemia. Antimicrob Agents Chemother. 2006;50:2541-3. http://dx.doi.org/10.1128/AAC.00224-06

18. Lazcano-Ponce E, Salazar-Martínez E, HernándezÁvila M. Case-control epidemiological studies: Theoretical bases, variants and applications. Salud Pública Mex. 2001;43:135-50.

19. Mikura S, Wada H, Okazaki M, Nakamura M, Honda K, Yasutake T, et al. Risk factors for bacteraemia attributable to Pseudomonas aeruginosa resistant to imipenem, levofloxacin, or gentamicin. J Hosp Infect. 2011;79:267-8. http://dx.doi.org/ 10.1016/j.jhin.2011.07.003
20. Voor In't Holt AF, Severin JA, Lesaffre EM, Vos MC. A systematic review and meta-analyses show that carbapenem use and medical devices are the leading risk factors for carbapenem-resistant Pseudomonas aeruginosa. Antimicrob Agents Chemother. 2014;58:2626-37. http://dx. doi.org/10.1128/AAC.01758-13

21. Echeverri-Toro LM, Rueda ZV, Maya W, Agudelo Y, Ospina S. Multidrug-resistant Klebsiella pneumoniae, predisposing factors and associated mortality in a tertiarycare hospital in Colombia. Rev Chilena Infectol. 2012;29:17582. http://dx.doi.org/10.4067/S0716-10182012000200009

22. Masterton RG. Antibiotic de-escalation. Crit Care Clin. 2011;27:149-62. http://dx.doi.org/10.1016/j.ccc.2010.09.009

23. Rodríguez-Osorio CA, Sánchez-Martínez CO, AraújoMeléndez J, Criollo E, Macías-Hernández AE, Poncede-León $\mathbf{A}$, et al. Impact of ertapenem on antimicrobial resistance in a sentinel group of Gram-negative bacilli: A 6 year antimicrobial resistance surveillance study. J Antimicrob Chemother. 2015;70:914-21. http://dx.doi.org/10. 1093/jac/dku471

24. Sousa D, Castelo-Corral L, Gutiérrez-Urbón JM, Molina F, López-Calviño B, Bou G, et al. Impact of ertapenem use on Pseudomonas aeruginosa and Acinetobacter baumannii imipenem susceptibility rates: Collateral damage or positive effect on hospital ecology? J Antimicrob Chemother. 2013;68:1917-25. http://dx.doi.org/10.1093/jac/dkt091

25. Sligl W, Taylor G, Brindley PG. Five years of nosocomial Gram-negative bacteriemia in a general intensive care unit: Epidemiology, antimicrobial susceptibility patterns, and outcomes. Int J Infect Dis. 2006;10:320-5. http://dx.doi. org/10.1016/j.ijid.2005.07.003

26. Morita Y, Tomida J, Kawamura Y. Responses of Pseudomonas aeruginosa to antimicrobials. Front Microbiol. 2014;4:422. http://dx.doi.org/10.3389/fmicb.2013.00422

27. Lin KY, Lauderdale TL, Wang JT, Chang SC. Carbapenemresistant Pseudomonas aeruginosa in Taiwan: Prevalence, risk factors, and impact on outcome of infections. J Microbiol Immunol Infect. 2014. http://dx.doi.org/10.1016/j. jmii.2014.01.005

28. Talpaert MJ, Gopal RG, Cooper BS, Wade P. Impact of guidelines and enhanced antibiotic stewardship on reducing broad-spectrum antibiotic usage and its effect on incidence of Clostridium difficile infection. J Antimicrob Chemother. $2011 ; 66: 2168-74$. http://dx.doi.org/10.1093/jac/dkr253

29. Leekha S, Terrell L, Edson RS. General principles of antimicrobial therapy. Mayo Clin Proc. 2011;86:156-67. http://dx.doi.org/10.4065/mcp.2010.0639

30. Swartz MN. Impact of antimicrobial agents and chemotherapy from 1972 to 1998 . Antimicrob Agents Chemother. 2000;44:2009-16. http://dx.doi.org/10.1128/AAC.44.8.20092016.2000

31. Zaloga GP. Parenteral nutrition in adult inpatients with functioning gastrointestinal tracts: Assessment of outcomes. Lancet. 2006;367:1101-11. http://dx.doi.org/10.1016/S01406736(06)68307-4

32. Turpin RS, Canada T, Rosenthal V, Nitzki-George D, Liu FX, Mercaldi CJ. Bloodstream infections associated with parenteral nutrition preparation methods in the United States: A retrospective, large database analysis. J Parenter Enteral Nutr. 2012;36:169-76. http://dx.doi. org/10.1177/0148607111414714 
33. Heuer JG, Sharma GR, Zhang T, Ding C, Bailey DL, Stephens EJ, et al. Effects of hyperglycemia and insulin therapy on outcome in a hyperglycemic septic model of critical illness. J Trauma. 2006;60:865-72. http://dx.doi. org/10.1097/01.ta.0000215565.29846.ab

34. Dissanaike S, Shelton M, Warner K, O'Keefe GE. The risk for bloodstream infections is associated with increased parenteral caloric intake in patients receiving parenteral nutrition. Crit Care. 2007;11:R114. http://dx.doi.org/10.1186/ cc6167

35. Turpin RS, Canada T, Rosenthal V, Nitzki-George D, Liu FX, Mercaldi CJ. Bloodstream infections associated with parenteral nutrition preparation methods in the
United States: A retrospective, large database analysis. J Parenter Enteral Nutr. 2012;36:169-76. http://dx.doi.org/10. $1177 / 0148607111414714$

36. Tuon F, Gortz L, Rocha J. Risk factors for pan-resistant Pseudomonas aeruginosa bacteriemia and the adequacy of antibiotic therapy. Braz J Infect Dis. 2012;16:351-6. http:// dx.doi.org/10.1016/j.bjid.2012.06.009

37. González AL, Leal AL, Cortés JA, Sánchez R, Barrero LI, Castillo JS, et al. Effect of adequate initial antimicrobial therapy on mortality in critical patients with Pseudomonas aeruginosa bacteriemia. Biomédica. 2014;34(Suppl.1):5866. http://dx.doi.org/10.1590/S0120-41572014000500008. 